\title{
Tumores raros en pediatría. Primer reporte en la Argentina
}

\author{
Rare tumors in pediatrics. First report in Argentina
}

\author{
Dra. Alejandra Casanovas ${ }^{a}$, Dra. Marianela Viso ${ }^{b}$, Dr. Guido Felizziab, \\ Dra. Adriana Rose $e^{b}$ Dra. Celeste Mattone ${ }^{c}$, Dra. Natalia Gazekc, Dra. Bettina Cervinid, \\ Dr. Carlos Giuseppucci ${ }^{e}$, Dra. Mariana Álvarez ${ }^{f}$ y Dr. Walter Cacciavillano ${ }^{b}$
}

a. Servicio de Oncología, Hospital General de Niños Pedro de Elizalde.

b. Servicio de Oncología, Hospital de Pediatría "Prof. Dr. Juan P. Garrahan".

c. Servicio de Endocrinología, CONICET fellow, Hospital de Pediatría "Prof. Dr. Juan P. Garrahan".

d. Servicio de Dermatología, Hospital de Pediatría "Prof. Dr. Juan P. Garrahan".

e. Servicio de Cirugía, Hospital de Pediatría "Prof. Dr. Juan P. Garrahan".

f. Servicio de Patología, Hospital de Pediatría "Prof. Dr. Juan P. Garrahan".

Ciudad Autónoma de Buenos Aires. Argentina.

\section{Correspondencia: \\ Dra. Alejandra Casanovas: alito1409@gmail.com}

\section{Financiamiento:} Este trabajo se realizó en el marco de una Beca en Oncopediatría otorgada por el Instituto Nacional del Cáncer.

Conflicto de intereses: ninguno que declarar.

Recibido: 11-12-2020

Aceptado: 28-5-2021

Publicado Primero en Internet: 15-10-2021

\section{RESUMEN}

Introducción. Los ensayos clínicos cooperativos han aumentado el conocimiento sobre los tumores pediátricos; sin embargo, no es el caso de los tumores raros (TR).

Objetivo. Describir prevalencia, características clínicas y evolución de los TRen la edad pediátrica diagnosticados en el Hospital Garrahan.

Material y métodos. Estudio descriptivo retrospectivo de pacientes entre 0 y 18 años con diagnóstico de TR ingresados entre enero de 2007 y diciembre de 2017.

Resultados. De 1657 pacientes con diagnóstico de tumores sólidos, 164 (9,9\%) correspondieron a TR, 71,95\% (118) eran menores de 14 años y $81,7 \%$ (130)eran varones. En orden de frecuencia, los TR fueron: carcinoma de tiroides (60), carcinoma suprarrenal (14), tumores pulmonares (14), melanoma (13), carcinoma de glándulas salivales (11), tumores gastrointestinales (8), tumores gonadales no germinales (7), tumores pancreáticos (7), carcinomas renales (6), carcinomas nasofaríngeos (5), feocromocitoma y paraganglioma (5) y carcinoma de timo en 1 paciente. El tratamiento recibido dependió del tipo de tumor y del estadio. Con una mediana de seguimiento de 34,9 meses (rango: $1-128,5$ meses), 133 pacientes ( $78,7 \%$ ) están vivos y solo 10 pacientes $(6 \%)$ se perdieron durante el seguimiento.

Conclusión. La prevalencia de TR fue del 9,9\%.El $27 \%$ se presentaron en adolescentes. Los tumores más frecuentemente diagnosticados fueron carcinoma de tiroides, carcinoma suprarrenal y melanoma. El tratamiento y la evolución varió según el tipohistológico. Se hallaron alteraciones moleculares predisponentes en el 5,3\% de los pacientes, el 3,5\% tenían antecedente de patología oncológica.

Palabras clave: neoplasias, enfermedades raras, niño, adolescente.

http:/ / dx.doi.org/10.5546/aap.2021.401

Texto completo en inglés:

http:/ / dx.doi.org/10.5546/ aap.2021.eng.401

Cómo citar: Casanovas A, Viso M, Felizzia G, Rose A, et al. Tumores raros en pediatría. Primer reporte en la Argentina. Arch Argent Pediatr 2021;119(6):401-407.

\section{INTRODUCCIÓN}

El cáncer en pediatría es poco frecuente. Su incidencia se estima en 1 en 7000 niños menores de 15 años, con más de 12000 casos nuevos por año en pacientes menores a 20 años en los Estados Unidos. ${ }^{1}$ La incidencia promedio en Argentina es de 1290 casos por año. ${ }^{2}$

Los tumores raros en pediatría se definen como "cualquier neoplasia maligna sólida caracterizada por una incidencia anual menor a 2/1000 000, que no se encuentra incluida en ningún protocolo de investigación". ${ }^{3}$ En la Clasificación Internacional de Cáncer Pediátrico (ICCC, por su sigla en inglés) ${ }^{4}$ se encuentran englobadas en los grupos XI (carcinomas corticosuprarrenal, tiroides, nasofaríngeo, melanoma y otros carcinomas no especificados), XII (tumores varios específicos e inespecíficos) y VI (tumores renales), donde se encuentran los carcinomas renales. Tienen como características una prevalencia baja en menores de 5 años (a excepción de los carcinomas corticosuprarrenales), predominan en adultos, son de origen epitelial y, dado el bajo número de pacientes, es difícil realizar protocolos de investigación.

El Registro Oncopediátrico Hospitalario Argentino ( $\mathrm{ROHA}$ ) utiliza para la codificación de la morfología y topografía los sistemas de clasificación de la Clasificación Internacional de Enfermedades Oncológicas (ICD-O-3, por su sigla en inglés $)^{5}$ y la ICCC. ${ }^{4}$ Según datos de este registro, en nuestro país se diagnostican 1290 pacientes por año en menores de 15 años, de los cuales 367 tienen neoplasias no 
hematopoyéticas extracraneales. ${ }^{2} \mathrm{El} 50 \%$ se atiende en el Hospital de Pediatría "Prof. Dr. Juan P. Garrahan".

El objetivo del presente trabajo es describir la prevalencia y las características de los tumores raros en la edad pediátrica diagnosticados en el Hospital Garrahan, teniendo en cuenta la representatividad poblacional antes mencionada.

\section{MATERIAL Y MÉTODOS}

Estudio retrospectivo descriptivo de pacientes entre 0 y 18 años ingresados entre enero de 2007 y diciembre de 2017, con diagnóstico de tumor raro.

Se completó una base de datos consignando los datos filiatorios, la fecha de diagnóstico (fecha del informe histopatológico), el informe histopatológico, la presencia de síndrome o condición predisponente, los estudios moleculares realizados, el tratamiento recibido, el seguimiento y la evolución.

Este estudio cuenta con la aprobación del Comité de Ética del hospital, respeta y resguarda la confidencialidad de los pacientes, ya que no se mencionan datos personales en el desarrollo del trabajo.

\section{RESULTADOS}

En este período ingresaron en el hospital 4100 pacientes con diagnóstico de patología oncológica. Con diagnóstico de tumores sólidos ingresaron 1657 pacientes. De estos, 164 (9,9\%) tenían tumores raros (con un promedio de 16 pacientes nuevos / año). En la Tabla 1 se describe la distribución de los tumores por grupo etario y sexo.

Tabla 1. Distribución de tumores según edad y sexo

\begin{tabular}{|c|c|c|c|c|c|}
\hline Tumor & $\mathbf{N}(\%)$ & Mediana de edad (años) & 0-14 años & 15-18 años & $\operatorname{Sexo}(M / F)$ \\
\hline Carcinoma de tiroides & $60(36,5)$ & $\begin{array}{c}12,6 \\
(3,57-17,84)\end{array}$ & 45 & 15 & $29 / 31$ \\
\hline Carcinoma suprarrenal & $14(8,5)$ & $\begin{array}{c}2,8 \\
(0,54-15,7)\end{array}$ & 13 & 1 & $7 / 7$ \\
\hline \multicolumn{6}{|l|}{ Tumores pulmonares: } \\
\hline Blastoma pleuropulmonar & $4(2,4)$ & 2,8 & 4 & 0 & $1 / 3$ \\
\hline Carcinomas & $3(1,8)$ & 16 & 0 & 3 & $0 / 3$ \\
\hline Tumores miofibroblásticos & $7(4,2)$ & 5,6 & 5 & 2 & $4 / 3$ \\
\hline Melanoma & $13(7,9)$ & $\begin{array}{c}6,2 \\
(1,56-15,26)\end{array}$ & 11 & 2 & $6 / 7$ \\
\hline Tumores carcinoides & $12(7,3)$ & $\begin{array}{c}12,17 \\
(7,4-16,5)\end{array}$ & 10 & 3 & $7 / 5$ \\
\hline Carcinoma de glándulas salivales & $11(6,7)$ & $\begin{array}{c}13,3 \\
(1,5-14,8)\end{array}$ & 8 & 3 & $4 / 7$ \\
\hline \multicolumn{6}{|l|}{ Tumores gastrointestinales: } \\
\hline Carcinoma de colon & $4(2,4)$ & 15,1 & 0 & 4 & $2 / 2$ \\
\hline GIST & $3(1,8)$ & 15,25 & 1 & 2 & $2 / 1$ \\
\hline GANT & $1(0,6)$ & 14,55 & 0 & 1 & $0 / 1$ \\
\hline Tumores gonadales no germinales & $7(4,2)$ & $\begin{array}{c}13,7 \\
(0,15-15,5)\end{array}$ & 4 & 3 & $1 / 6$ \\
\hline \multicolumn{6}{|l|}{ Tumores pancreáticos: } \\
\hline Tumor de Frantz & $6(3,6)$ & 12,7 & 6 & 0 & $0 / 6$ \\
\hline Pancreatoblastoma & $1(0,6)$ & 5,4 & 1 & 0 & $1 / 0$ \\
\hline Carcinoma renal & $6(3,6)$ & $\begin{array}{c}9,1 \\
(5,8-14,3)\end{array}$ & 6 & 0 & $3 / 3$ \\
\hline Carcinoma nasofaríngeo & $5(3)$ & $\begin{array}{c}14,2 \\
(13,3-16,9)\end{array}$ & 2 & 3 & $3 / 2$ \\
\hline Feocromocitoma o paraganglioma & $5(3)$ & $\begin{array}{c}10,69 \\
(9,04-15,28)\end{array}$ & 3 & 2 & $3 / 2$ \\
\hline Carcinoma de timo & $1(0,6)$ & 11,5 & 1 & 0 & $1 / 0$ \\
\hline Total & 164 & 12,38 & 118 & 46 & $134 / 30$ \\
\hline
\end{tabular}

GIST: tumor del estroma gastrointestinal (por su sigla en inglés),

GANT: tumor gastrointestinal derivado del ganglio autonómico (por su sigla en inglés). 
Las distintas histologías de algunos tumores se describen en la Tabla 2, y en la Tabla 3 se describen las condiciones predisponentes de otros.

Los pacientes con diagnóstico de carcinoma de tiroides recibieron tratamiento con tiroidectomía y con iodo radioactivo en el servicio de Endocrinología. Con una mediana de seguimiento de 38,4 meses (rango 1-128 meses), ningún paciente presentó eventos. En el seguimiento, se perdieron 4 pacientes.
Los pacientes con diagnóstico de carcinoma suprarrenal se clasificaron, según el Grupo de Oncología Pediátrica (COG, por su sigla en inglés), en estadio I ( 2 pacientes), estadio II (2 pacientes), estadio III (5 pacientes) y estadio IV (5 pacientes). Los pacientes con tumores en estadios I y II recibieron tratamiento quirúrgico, mientras que 4 pacientes con tumores en estadio III y 3 en estadio IV recibieron quimioterapia (QMT). Para una mediana de seguimiento de 24,6 meses (rango: 1-66 meses), los pacientes

TABLA 2. Subtipo histológico de los tumores

\begin{tabular}{ll}
\hline Tumor (n pacientes) & Subtipo histológico (n pacientes) \\
\hline Carcinoma de tiroides (60) & Papilar (50) \\
& Folicular (7) \\
Carcinoma de glándulas salivales (11) & Medular (3) \\
& Mucoepidermoide (7) \\
& De células acinares (2) \\
Tumores gonadales no germinales (7) & Adenoideoquístico (1) \\
& Pobremente diferenciado (1) \\
& De la granulosa juvenil (3) \\
& Sertoli-Leydig (2) \\
Carcinoma renal (6) & Estromal esclerosante (1) \\
& Carcinoma de células pequeñas del ovario (1) \\
Feocromocitoma/paraganglioma (5) & Células claras (4) \\
& Papilar (1) \\
& Subtipo cromófobo (1) \\
& Feocromocitoma (2) \\
& Paraganglioma (2) \\
\hline
\end{tabular}

TABLA 3. Alteraciones genético-moleculares con predisposición al cáncer halladas en este estudio

\begin{tabular}{ll}
\hline Tumor (total de pacientes) & Alteración y número de pacientes \\
\hline Carcinoma de tiroides (60) & $\begin{array}{l}\text { Patología previa (8 pacientes): } 6 \text { desarrollaron el carcinoma de tiroides como } \\
\text { segunda neoplasia (2 con linfoma de Hodgkin que recibieron RDT en la } \\
\text { zona del cuello, } 1 \text { con antecedente de tumor de células de Sertoli-Leydig con } \\
\text { mutación del gen DICER-1, } 1 \text { con rabdomiosarcoma de cavum que recibió }\end{array}$ \\
RDT, 1 con diagnóstico de leucemia linfoblástica aguda e hipopituitarismo \\
con recaída testicular que recibió RDT, y 1 con hepatocarcinoma asociado a \\
glucogenosis de tipo I, que recibió un trasplante hepático). Un paciente tenía \\
antecedente de adenoma hipofisario y 1 tenía bocio nodular autoinmune.
\end{tabular}

RDT: radioterapia, ADN: ácido desoxirribonucleico. 
en estadio I y II se encuentran vivos y libres de enfermedad. Fallecieron 3 pacientes (estadio IV), uno al mes del diagnóstico, sin tratamiento, y los otros dos por progresión de enfermedad a los 6 y 30 meses del diagnóstico. Los pacientes que recibieron tratamiento adyuvante no recibieron mitotano, debido a que no se dispone de esta medicación en Argentina.

De los 14 pacientes con tumor pulmonar, 4 tuvieron diagnóstico de blastoma pleuropulmonar (2 de tipo I y 2 de tipo II). Estos últimos recibieron tratamiento adyuvante, uno con QMT y el otro con QMT y radioterapia (RDT), ambos fallecieron por progresión de enfermedad a los 4 y 6 meses del diagnóstico, respectivamente. La resección quirúrgica fue el tratamiento realizado en los blastomas de tipo I, encontrándose libres de enfermedad con una mediana de seguimiento de 24 meses (17 y 31 meses, respectivamente). Un paciente desarrolló un nefroma quístico 2 años después del diagnóstico inicial.

Siete pacientes fueron diagnosticados con tumor miofibroblástico inflamatorio, cuyo tratamiento fue exclusivamente quirúrgico. Dos pacientes presentaron recaída local, logrando una nueva remisión con cirugía, 1 paciente falleció a los 3 meses del diagnóstico por progresión, 2 pacientes se perdieron en el seguimiento y 4 (incluidos los dos recaídos) se encuentran vivos sin enfermedad a más de 5 años del diagnóstico.

Por último, 3 pacientes tuvieron diagnóstico de carcinoma (epidermoide, mioepitelial y adenoideo-quístico). El paciente con carcinoma mioepitelial fue operado y seguido en este hospital, los otros 2 pacientes fueron derivados a otra institución. El paciente con carcinoma epidermoide recibió QMT adyuvante y falleció por progresión de enfermedad a los 15 meses del diagnóstico. Los otros dos pacientes se encuentran vivos, sin enfermedad, a 25 y 63 meses del diagnóstico, respectivamente.

De los 14 pacientes con diagnóstico de melanoma, 8 continúan vivos y libres de enfermedad, con una mediana de seguimiento de 26 meses (rango 2-89 meses). Cuatro pacientes fallecieron por progresión de enfermedad a los 2 , 4, 19 y 26 meses del diagnóstico, respectivamente. Dos pacientes se perdieron en el seguimiento.

Los 12 pacientes con tumores carcinoides (5 de pulmón y 7 de apéndice cecal) recibieron solo tratamiento quirúrgico. Actualmente se encuentran vivos, sin enfermedad, luego de una mediana de seguimiento de 35 meses (rango 3-71 meses).

De los 11 pacientes con diagnóstico de carcinoma de glándulas salivales, solo el paciente con carcinoma pobremente diferenciado se presentó con metástasis y fue el único que recibió tratamiento adyuvante (QMT y RDT), falleciendo por progresión de enfermedad a los 8 meses del diagnóstico. Un paciente con carcinoma mucoepidermoide presentó recaída local a los 55 meses del diagnóstico, logrando una nueva remisión con cirugía. Nueve pacientes se encuentran vivos sin enfermedad, con una mediana de seguimiento de 29 meses (rango 2-79 meses). Un paciente se perdió en el seguimiento.

Los 4 pacientes con carcinoma de colon presentaron metástasis al diagnóstico y recibieron QMT, 1 de ellos derivado a otra institución. Todos fallecieron por progresión de la enfermedad, con una mediana de sobrevida desde el diagnóstico de 6,7 meses (rango 5-11 meses).

Los pacientes con diagnóstico de tumor del estroma gastrointestinal (GIST, por su sigla en inglés) (3 pacientes) y tumor gastrointestinal derivado del ganglio autonómico (GANT, por su sigla en inglés) (1 paciente) recibieron solo tratamiento quirúrgico. Un paciente con diagnóstico de GIST presentó recidiva locorregional a los 24 meses del diagnóstico, recibió imatinib durante dos años permaneciendo estable y se encuentra vivo a 79 meses del diagnóstico. Los otros 3 pacientes se encuentran vivos sin enfermedad, con un seguimiento de entre 5 y 20 meses.

Los 7 pacientes con tumores gonadales no germinales fueron clasificados, luego de la cirugía, como estadio 1 (3 pacientes) y II (4 pacientes) según la clasificación postquirúrgica del Grupo Internacional de Rabdomiosarcoma (IRS, por su sigla en inglés). Solo el carcinoma de células pequeñas de ovario fue metastásico. Cinco pacientes realizaron tratamiento, 4 QMT (1 recayó) y 1 solo (carcinoma de células pequeñas) recibió QMT y RDT, y falleció a los 3 meses del diagnóstico por progresión de enfermedad. El resto de los pacientes se encuentran vivos y sin enfermedad con una mediana de seguimiento de 18 meses (rango: 8-119 meses).

Seis pacientes presentaron tumor sólido seudopapilar del páncreas (tumor de Frantz) y solo recibieron tratamiento quirúrgico. Un paciente presentó pancreatoblastoma localizado y este recibió QMT (con cisplatino y doxorrubicina), finalizando el tratamiento en remisión completa. Se encuentra vivo y sin enfermedad a 7 meses del diagnóstico.

De los 6 pacientes con diagnóstico de carcinoma renal, se realizó una nefrectomía en cinco. Tres pacientes presentaron compromiso ganglionar, ninguno con metástasis en el momento del diagnóstico. Dos pacientes 
recibieron QMT y RDT, 1 paciente realizó RDT y el resto sólo recibió tratamiento quirúrgico. El paciente que fue biopsiado no recibió tratamiento y falleció a los 21 meses del diagnóstico. Un paciente presentó una recaída metastásica a los 5 meses del diagnóstico, y falleció 6 meses después. Los otros 4 pacientes se encuentran vivos y sin enfermedad, con una mediana de seguimiento de 52 meses (rango: 18-86 meses).

De los pacientes con diagnóstico de carcinoma nasofaríngeo, 3 tenían metástasis al momento del diagnóstico. Todos realizaron tratamiento (QMT + RDT 4, uno recibió solo RDT). Dos pacientes recayeron con metástasis, uno falleció a los 33 meses del diagnóstico. El resto de los pacientes se encuentran vivos y libres de enfermedad, con una mediana de seguimiento de 60 meses (rango: 26-105 meses).

Los pacientes con diagnóstico de feocromocitoma o paraganglioma fueron intervenidos quirúrgicamente. Un paciente presentó metástasis al diagnóstico y falleció a los 50 meses del diagnóstico. Tres se encuentran sin enfermedad, con una mediana de seguimiento de 60 meses (rango: 22-53 meses), y uno se perdió en el seguimiento.

El paciente con diagnóstico de carcinoma de timo presentó metástasis. La pieza resecada tenía márgenes comprometidos. Luego de la cirugía, realizó QMT y RDT, falleció a los 7 meses del diagnóstico por progresión de la enfermedad.

No hubo ningún paciente con diagnóstico de carcinoma de mama.

\section{DISCUSIÓN}

El presente trabajo comprende el primer reporte de tumores raros de Argentina. Si bien fue realizado en una sola institución, esta recibe el $50 \%$ de los tumores sólidos del país. Se fortalece el concepto de representatividad al comparar el total de pacientes con estos diagnósticos registrados en el $\mathrm{ROHA}^{2}$ entre los años 2000-2013, con un promedio de 30 pacientes por año, con el presente estudio realizado durante 10 años, en el que el promedio es de 16 pacientes por año.

Al tratarse de un estudio retrospectivo, donde los pacientes recibieron tratamiento en diferentes servicios del hospital, por lo que es probable que el número de pacientes registrados sea menor al real. Esto se transforma, así, en una de las principales debilidades del estudio, similar a lo observado en el primer reporte del grupo de Tumores Raros en la Edad Pediátrica (TREP, por su sigla en italiano), ${ }^{3}$ quienes, retrospectivamente, entre los años 1982-1998, describen una frecuencia de 15 pacientes nuevos por año y, al realizar el estudio de manera prospectiva, el número asciende a 49 pacientes por año (entre enero de 2000 y diciembre de 2005). Otro dato es que, mientras para el grupo italiano y para el COG los tumores raros corresponden entre un $8 \%$ y un $10 \%$ de todos los tumores diagnosticados en la edad pediátrica, ${ }^{3,6}$ en nuestra cohorte, de 4100 pacientes con patología oncológica ingresados, 164 tenían tumores raros (4\%).

Según datos del Programa de Resultados Finales de Vigilancia Epidemiológica (SEER, por su sigla en inglés), ${ }^{7}$ el $75 \%$ de los tumores raros ocurren en pacientes adolescentes y adultos jóvenes. En nuestra cohorte, los pacientes mayores de 15 años representan el $27 \%$, porcentaje similar al encontrado por el grupo italiano $(22 \%$ de pacientes entre 15 y 17 años $^{3}$ ). Es probable que esto se deba a que los pacientes diagnosticados con estos tumores en la adolescencia sean manejados en centros de adultos.

En nuestra serie, los tumores más frecuentemente diagnosticados fueron carcinoma de tiroides, carcinoma suprarrenal y melanoma. Si se los separa por grupos etarios, en niños (014 años) se respeta el mismo orden de frecuencia, mientras que, en adolescentes (15-18 años), el carcinoma de tiroides continúa en primer lugar en frecuencia, mientras que el segundo lugar lo ocupa el carcinoma de colon. El resto tuvo una distribución similar. Cabe destacar que los pacientes con diagnóstico de carcinoma de tiroides son seguidos y tratados solo por el servicio de Endocrinología del hospital, excepto aquellos con neoplasia previa. Algo similar ocurre con los pacientes con diagnóstico de melanoma, cuyo servicio de referencia es el de Dermatología, y raras veces se atienden en el servicio de Oncología. Esta es una limitación que se observa al momento de registrar a los pacientes, compartida por otros grupos internacionales. . $^{3-6-8}$

Algunos de estos tumores raros cuentan con la particularidad de tener alteraciones moleculares específicas. El conocimiento de la presencia de estas es un dato fundamental para un consejo genético adecuado y ofrece la oportunidad de estudiar a los familiares del caso índice para un eventual diagnóstico precoz. En el presente estudio, se encontró que sólo se estudió al $43 \%$ de los pacientes con diagnóstico de carcinoma suprarrenal en busca de una mutación del gen p53, que estaba alterado en la mitad de los casos. Sería importante conocer el estado de este gen, dado que se sabe que hay una mayor incidencia de este tumor en el sur de Brasil por una mutación específica, ${ }^{9-10}$ y que en el hospital se atiende un gran número de pacientes provenientes de provincias limítrofes con ese país.

La enfermedad de Von Hippel-Lindau es 
un síndrome familiar con predisposición al cáncer, asociado a una variedad de tumores benignos y malignos, principalmente tumores de retina y de cerebelo, y al hemangioblastoma espinal, carcinoma de células renales y, en mucha menor frecuencia, feocromocitoma. ${ }^{11}$ Se debe a la mutación del gen $V H L$, ubicado en el cromosoma 3p25.3; se hereda de manera autosómica dominante y su detección en uno de los pacientes con feocromocitoma no solo es útil para realizar consejo genético, sino para realizar controles periódicos con el objetivo de descartar el resto de los tumores que aparecen con mayor frecuencia en este síndrome.

Otra mutación para investigar es compartida por dos patologías, la del gen DICER-1 (14q32.13). Esta suele asociarse con blastomas pleuropulmonares, tumores del estroma de los cordones sexuales de ovario y nefromas quísticos, entre otros. ${ }^{12}$ Entre los pacientes de esta serie con diagnóstico de blastoma pleuropulmonar, solo se estudiaron aquellos con tumores de tipos I: uno de ellos desarrolló posteriormente un nefroma quístico, en el cual no se encontró la mutación, y en el otro se encuentra pendiente el resultado. Con respecto a los pacientes con diagnóstico de tumores gonadales no germinales, esta mutación se encontró en las dos pacientes con diagnóstico de tumor de células de Sertoli-Leydig, descripto en la bibliografía como la histología más frecuente para esta mutación.

Al recopilar datos con respecto a las alteraciones moleculares en pacientes aquí presentados, se encontró que no se realizó el estudio en todos ellos, en su mayoría por la falta de disponibilidad en el momento del diagnóstico.

Un dato relevante encontrado en este estudio es que el $10 \%$ de los pacientes con diagnóstico de carcinoma de tiroides tenían historia de otra patología oncológica previa. Cuatro de ellos habían recibido RDT (2 específicamente en la zona del cuello), antecedente que aumenta un $17 \%$ el riesgo de padecer carcinoma de tiroides, ya que la glándula es muy vulnerable a los efectos carcinogénicos de las radiaciones. ${ }^{13} \mathrm{Un}$ paciente recibió tratamiento inmunosupresor por haber recibido un trasplante y en otro se detectó una mutación en el gen DICER-1. Este hallazgo refuerza la importancia del seguimiento y control postratamiento de los pacientes oncológicos.

Con respecto al seguimiento de los pacientes con diagnóstico de tumores raros, cabe destacar que solo un $6 \%$ se perdieron en el seguimiento.

Conocer estos datos iniciales permitirá proponer nuevas estrategias de registro y formular, en forma interdisciplinaria, propuestas de diagnóstico, tratamiento y seguimiento. La recopilación multicéntrica de información y en el nivel nacional es un desafío a realizar como siguiente paso.

\section{CONCLUSIÓN}

La prevalencia de TR fue del 9,9\% de los tumores sólidos. El $27 \%$ se presentaron en pacientes fueron adolescentes. Los tumores diagnosticados con mayor frecuencia fueron carcinoma de tiroides, carcinoma suprarrenal y melanoma. El tratamiento y la evolución varió según el tipo histológico. Se hallaron alteraciones moleculares predisponentes en el 5,3\% de los pacientes, y el 3,5 \% tenían historia de patología oncológica.

\section{REFERENCIAS}

1. Gurney JG, Bondy ML. Epidemiology of childhood cancer. In: Pizzo PA, Poplack DG (eds). Principles and practice of pediatric oncology. 5th ed. Philadelphia, PA: Lippincott Williams \& Wilkins; 2006.Págs.1-13.

2. Moreno F, Chaplin MA. Registro oncopediátrico hospitalario argentino. 6a ed. Ciudad Autónoma deBuenos Aires: Instituto Nacional del Cáncer; 2018.

3. Ferrari A, Bisogno G, De Salvo GL, Indolfi P, et al. The challenge of very rare tumours in childhood: The Italian TREP project. Eur J Cancer. 2007; 43(4):654-9.

4. International Classification of Childhood Cancer. Subgroup XI, of the SEER database 2003-2007. [Acceso: 1 de junio de 2021]. Disponible en: http://seer.cancer. gov/csr/1975_2007/results_merged/sect_29_childhood_ cancer_iccc.pdf

5. Fritz A, Perci C, Jack A, Shanmugaratnam K, et al (eds). ICD-O International classification of diseases for oncology. $3^{\text {rd }}$ ed. Geneva: World Health Organization; 2000.

6. Pappo AS, Krailo M, Chen Z, Rodríguez-Galindo C, et al. Infrequent tumor initiative of the Children's Oncology Group: Initial lessons learned and their impact on future plans. J Clin Oncol. 2010; 28(33):5011-6.

7. Ries L, Smith M, Gurney J, Linet M, et al (eds). Cancer Incidence and Survival among Children and Adolescents: United States SEER Program 1975-1995. Bethesda, MD: National Cancer Institute, SEER program NIH Pub No. 99-4649; 1999.

8. Villarroel M, Chantada G. Tumores raros en niños y adolescentes. Rev Med Clin Condes. 2015; 26(4):495-502.

9. Rodríguez-Galindo C, Pappo AS, Krailo MD, Pashankar F, et al. Treatment of childhood adrenocortical carcinoma (ACC) with surgery plus retroperitoneal lymph node dissection (RPLND) and multiagent chemotherapy: results of the Children's Oncology Group ARAR0332 protocol. J Clin Oncol. 2016; 34(Suppl 15):10515.

10. Ribeiro RC, Pinto E, Zambetti G, Rodríguez-Galindo C. The International Pediatric Adrenocortical Tumor Registry initiative: Contributions to clinical, biological, and treatment advances in pediatric adrenocortical tumors. Mol Cell Endocrinol. 2012; 351(1):37-43.

11. Varshney N, Kebede AA, Owusu-Dapaah H, Lather J, et al. A review of von Hippel-Lindau syndrome. J Kidney Cancer VHL. 2017; 4(3): 20-9. 
12. Schultz KA, Stewart DR, Kamihara J, Bauer AJ, et al. DICER1-Tumor predisposition. In: Adam M, Ardinger HH, Pagon RA, Wallace SE, et al (eds). GeneReviews. Seattle, WA: University of Washington; 2014.
13. Waguespack SG. Thyroid Sequelae of Pediatric Cancer Therapy. Horm Res Paediatr. 2018; 91(2):104-17.

\section{Artículos seleccionados}

Los siguientes resúmenes y comentarios de trabajos seleccionados se encuentran disponibles en la versión electrónica de este número.

JAMA Netw Open. 2021; 4 (3): e211816. doi: 10.1001 / jamanetworkopen.2021.1816

Disparidades raciales/étnicas en el parto muy prematuro y el parto prematuro antes y durante la pandemia de COVID-19 (Janevic T, et al. Racial/ethnic disparities in very preterm birth and preterm birth before and during the COVID-19 pandemic)

Comentario: Dr. José M. Ceriani Cernadas. Hospital Italiano de Buenos Aires.

Pediatr Pulmonol. 2021 Apr 5. doi: 10.1002/ppul.25391. Online ahead of print.

¿Hay un lugar para la bronquiolitis en la era COVID-19? Ausencia de hospitalizaciones por virus respiratorios comunes durante el invierno 2020 (Ferrero F, et al. Is there a place for bronchiolitis in the COVID-19 era? Lack of hospitalizations due to common respiratory viruses during the 2020 winter)

Comentario: Dr. Santiago Vidaurreta. Hospital Universitario Cemic. Ciudad de Buenos Aires.

Pediatr Pulmonol. 2021 Feb;56(2):486-494.

$\mathrm{CO}_{2}$ de final de espiración y $\mathrm{CO}_{2}$ trasncutáneo: ¿estamos listos para reemplazar el $\mathrm{CO}_{2}$ arterial en niños despiertos? (Dicembrino $\mathrm{M}$, et al. End-tidal $\mathrm{CO}_{2}^{2}$ and transctutaneous $\mathrm{CO}_{2}$ : Are we ready to replace arterial $\mathrm{CO}_{2}$ in awake children?)

Comentario: Dra. María José Guerdile. Hospital de Pediatría Prof. Dr. J. P. Garrahan. Ciudad de Buenos Aires.

JAMA Netw Open. 2021 Jun 1;4(6):e2111836.

Efecto de las pruebas rápidas de virus respiratorios sobre la prescripción de antibióticos en niños que se presentan al Servicio de Urgencias por enfermedad respiratoria aguda: ensayo clínico aleatorizado (Rao $S$, et al. Effect of rapid respiratory virus testing on antibiotic prescribing among children presenting to the emergency department with acute respiratory illness: a randomized clinical trial)

Comentario: Dra. Laura Beatriz Moreno. Hospital de Niños Santísima Trinidad de Córdoba.

BMJ Qual Saf. 2021 Apr 23:bmjqs-2020-012370.

Implementación de un programa de mejora en el traspaso de pacientes y reducción de eventos adversos en unidades de terapia intensiva pediátrica de Argentina: estudio escalonado (Jorro-Barón F, et al. Handoff improvement and adverse event reduction programme implementation in paediatric intensive care units in Argentina: a stepped-wedge trial) Comentario: Dr. Cristian García Roig. Sanatorio Mater Dei. Ciudad de Buenos Aires.

Paediatr Drugs. 2021 May;23(3):223-240.

Intervenciones para reducir los errores de prescripción pediátrica en entornos de atención médica profesional: una revisión sistemática de la última década (Koeck JA, et al. Interventions to reduce pediatric prescribing errors in professional healthcare settings: a systematic review of the last decade)

Comentario: Dr. José M. Ceriani Cernadas. Hospital Italiano de Buenos Aires.

PLoS One. 2021 May 20;16(5):e0251496.

Impacto de la vacuna de la varicela en los brotes nosocomiales y el manejo del seguimiento de la profilaxis postexposición en un hospital pediátrico (Gentile A, et al. Impact of varicella vaccine on nosocomial outbreaks and management of post exposure prophylaxis following in a paediatric hospital)

Comentario: Dra. Gabriela Ensinck. Hospital de Niños Vilela Rosario. Santa Fe.

Pediatrics. 2021 Aug;148(2):e2020049411.

Discusiones sobre salud sexual y reproductiva durante los controles en salud (Sieving RE, et al. Sexual and reproductive health discussions during preventive visits)

Comentario: Dr. Gonzalo Agüero. Hospital de Agudos Dr. Cosme Argerich. Ciudad de Buenos Aires.

Lancet Infect Dis. 2021 Jul;21(7):993-1003.

Efectos no específicos inducidos por la BCG en otras infecciones en recién nacidos de Uganda: estudio aleatorizado controlado ciego (Prentice S, et al. BCG-induced non-specific effects on heterologous infectious disease in Ugandan neonates: an investigator-blind randomised controlled trial)

Comentario: Dra. Norma E. González. Hospital General de Niños Pedro de Elizalde. Ciudad de Buenos Aires. 